\title{
Incidental extraspinal findings on magnetic resonance imaging of intervertebral discs
}

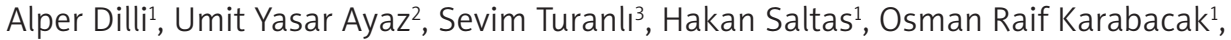 \\ Cagrı Damar ${ }^{1}$, Baki Hekimoglu ${ }^{1}$
}

${ }^{1}$ Ministry of Health, Dıskapı Yıldırım Beyazıt Training and Research Hospital, Ankara,
Turkey
${ }^{2}$ Ministry of Health, Mersin Women's and Children's Hospital, Mersin, Turkey
${ }^{3}$ Ministry of Health, Ankara Oncology Education and Research Hospital, Ankara, Turkey

Submitted: 12 December 2011

Accepted: 28 June 2012

Arch Med Sci 2014; 10, 4: 757-763

DOI: 10.5114/aoms.2014.44868

Copyright (c 2014 Termedia \& Banach

\begin{abstract}
Introduction: We aimed to evaluate pathological extraspinal findings and congenital anomalies/anatomical variations that were incidentally detected on the magnetic resonance imaging (MRI) scans of intervertebral discs, to find the frequencies of these incidental findings, and to emphasise the clinical importance of them.

Material and methods: A retrospective study including 1031 consecutive patients (730 females and 301 males, with a median age of 46 years) was conducted by evaluating a total of $1106 \mathrm{MRI}$ examinations of intervertebral discs. Examinations were performed with a $1.5 \mathrm{~T} \mathrm{MRI} \mathrm{unit.} \mathrm{Incidental} \mathrm{findings} \mathrm{were} \mathrm{classified} \mathrm{as}$ pathological findings and congenital anomalies/anatomical variations.

Results: The percentages of incidental extraspinal pathological findings and congenital anomalies/anatomical variations were $16.6 \%$ (95\% confidence interval (Cl): $14.4-18.8)$ and $3.7 \%(95 \% \mathrm{Cl}: 2.6-4.3)$, respectively. The percentage of incidental extraspinal pathological findings on cervical spinal MRI was $25.7 \%$ (95\% Cl: 20.1-31.7), thyroid nodules being the most common incidental findings. On thoracic spinal MRI $(n=19)$, inferior pole thyroid nodules were demonstrated as incidental extraspinal pathological findings, with a percentage of $10.5 \%$ (95\% Cl: 9.6-11.5). On lumbar spinal MRI, incidental pathological findings were detected with a percentage of $14.2 \%(95 \% \mathrm{Cl}$ : 11.9-16.6), while the percentage of congenital anomalies/anatomical variations was $4.8 \%$ (95\% Cl: 3.4-6.3). Eventually, 6.5\% (95\% Cl: $2.6-9.4)$ of all cases with incidental extraspinal pathological findings underwent surgery. Conclusions: On MRI examination of intervertebral discs, paying attention to incidentally detected pathological extraspinal findings and congenital anomalies/anatomical variations is very important due to the fact that they can alter the treatment of the patient or affect the patient's life.
\end{abstract}

Key words: magnetic resonance imaging, intervertebral disc, incidental findings, congenital anomalies.

\section{Introduction}

Back pain is one of the most common health problems in developed countries [1-4]. Different imaging modalities have been used in patients with back pain and neurological disorders. Plain radiography and computed tomography (CT) have a serious burden of ionising-radiation for the patient in various doses $[3,5,6]$. Magnetic resonance imaging (MRI)
Corresponding author: Dr. Alper Dilli

Department of Radiology Diskapı Yildirim Beyazit Training and Research Hospital Ministry of Health 06110 Dıskapı/Ankara, Turkey Phone: +905304653941 E-mail: alperdilli@yahoo.com 
provides multiplanary, non-ionising imaging for both bone and soft tissues, and it has become the most validated and preferred imaging modality due to the fact that it permits extraspinal evaluation [7]. In the course of a 12-year interval, use of lumbar MRI was reported to have increased by $307 \%$ [8]. Concordantly, the frequency of detecting incidental extraspinal findings has also increased $[2,3,9]$. Incidental findings were detected in $8.4 \%$ of patients who underwent spine lumbar MRI [10]. By reporting these findings properly and making a correct, definitive diagnosis, the radiologist will prevent unnecessary invasive operations and additional examinations for the patient. However, not reporting them may have clinical importance to a degree that can affect the patient's life severely, particularly in cases such as recurrence of a renal cell carcinoma. The main purpose of this study was to evaluate pathological extraspinal findings and congenital anomalies/anatomical variations which were incidentally detected on the MRI of intervertebral discs in patients who were referred with complaints of pain and neurological disorders, to find the frequencies of these incidental findings and to emphasise the clinical importance of them.

\section{Material and methods}

\section{Procedures related to patient selection and follow-up}

Between October 2009 and February 2010, a retrospective study including 1031 consecutive patients (730 females and 301 males with a median age of 46 years) was conducted by evaluating a total of $1106 \mathrm{MRI}$ examinations of intervertebral discs. The patients who gave any information of a previously known extraspinal pathology (extraspinal masses, cancer, etc.) were excluded. Of these, 237 were cervical MRI, 19 were thoracic MRI, and 850 were lumbar MRI. There were $730 \mathrm{fe}$ males and 301 males with a median age of 46 years (range, 8-82 years), with $95 \% \mathrm{Cl}$ of $44.9-47.0$ years. This study was performed according to the World Medical Association Declaration of Helsinki. Incidental findings were classified as pathological findings and congenital anomalies/anatomical variations. Congenital anomaly was defined as an abnormal physical condition resulting from defective genes or developmental deficiencies, whereas anatomical variation was defined as marked difference or deviation from the normal or recognised form, function, or structure. When a pathology or congenital anomaly/anatomic variation was detected on a spinal MRI, the referring physicians were informed and they, in turn, undertook the follow-up of the patient. The patients who underwent an invasive procedure (biopsy, surgery) were recorded.

\section{MR Imaging technique}

Examinations were performed with a 1.5 T MRI unit (Philips Achiva, Philips Medical Systems, Eindhoven, Netherlands) with spine coil, in supine position. Cervical spinal MRI protocol included sagittal T1-weighted turbo spin-echo (TSE) images (TR/TE, 400/9 ms; slice thickness/interslice gap, 4/ $0.4 \mathrm{~mm}$ and NEX, 3), sagittal T2-weighted TSE images (TR/TE, 3000/120 ms; slice thickness/ interslice gap, 4/0.4 mm and NEX, 3) and axial T2-weighted GE, FFE images (TR/TE, 600/14 ms; slice thickness/interslice gap, 4/0.4 mm, flip angle $25^{\circ}$ and NEX, 3). Thoracic spinal MRI protocol included sagittal T1-weighted TSE images (TR/ TE, 400/9 ms; slice thickness/interslice gap, 4/0.4 $\mathrm{mm}$; NEX, 3), sagittal T2-weighted TSE images (TR/TE, 3000/120 ms; slice thickness/interslice gap, 4/0.4 mm; NEX, 3), and axial T2-weighted TSE images (TR/TE, 4000/120 ms; slice thickness/ interslice gap, 4/0.4 mm; NEX, 3). Lumbar spinal MRI protocol included sagittal T1-weighted TSE images (TR/TE, 400/9 ms; slice thickness/interslice gap, 4/0.4 mm; NEX, 3), sagittal T2-weighted TSE images (TR/TE, 3000/120 ms; slice thickness/interslice gap, 4/0.4 mm; NEX, 3), and axial T2-weighted TSE images (TR/TE, 3000/110 ms; slice thickness/interslice gap, 4/0.4 mm; NEX, 3). On cervical spinal MRI, thyroid glands, parathyroid glands or their probable locations, paranasal sinuses, cerebellum and other posterior fossa structures, vascular structures, and lymph nodes of the neck were evaluated. On thoracic spinal MRI, lung parenchyma, mediastinum, pleura, lymph nodes, posterior ribs, and adrenal glands were evaluated. On lumbar spinal MRI, kidneys, adrenal glands, liver, lymph nodes, and vascular structures (inferior vena cava, abdominal aorta, renal veins and arteries, and other vasculatures) were evaluated.

\section{Image analysis}

Spinal MRI (cervical, thoracic, and lumbar spinal) images were evaluated by three radiology specialists, each with at least 5 years experience. One of the specialists had expertise in head and neck radiology, one in thorax radiology, and the other in abdomen radiology. The images were evaluated on the same computer. We used a picture archiving and communication system (PACS), and reported the pathologies, congenital anomalies, and anatomical variations, all of which could be detected during the interpretation of the images.

\section{Statistical analysis}

Descriptive statistics were calculated for incidental extraspinal findings in the neck, thorax, and abdomen. All analyses were carried out using 

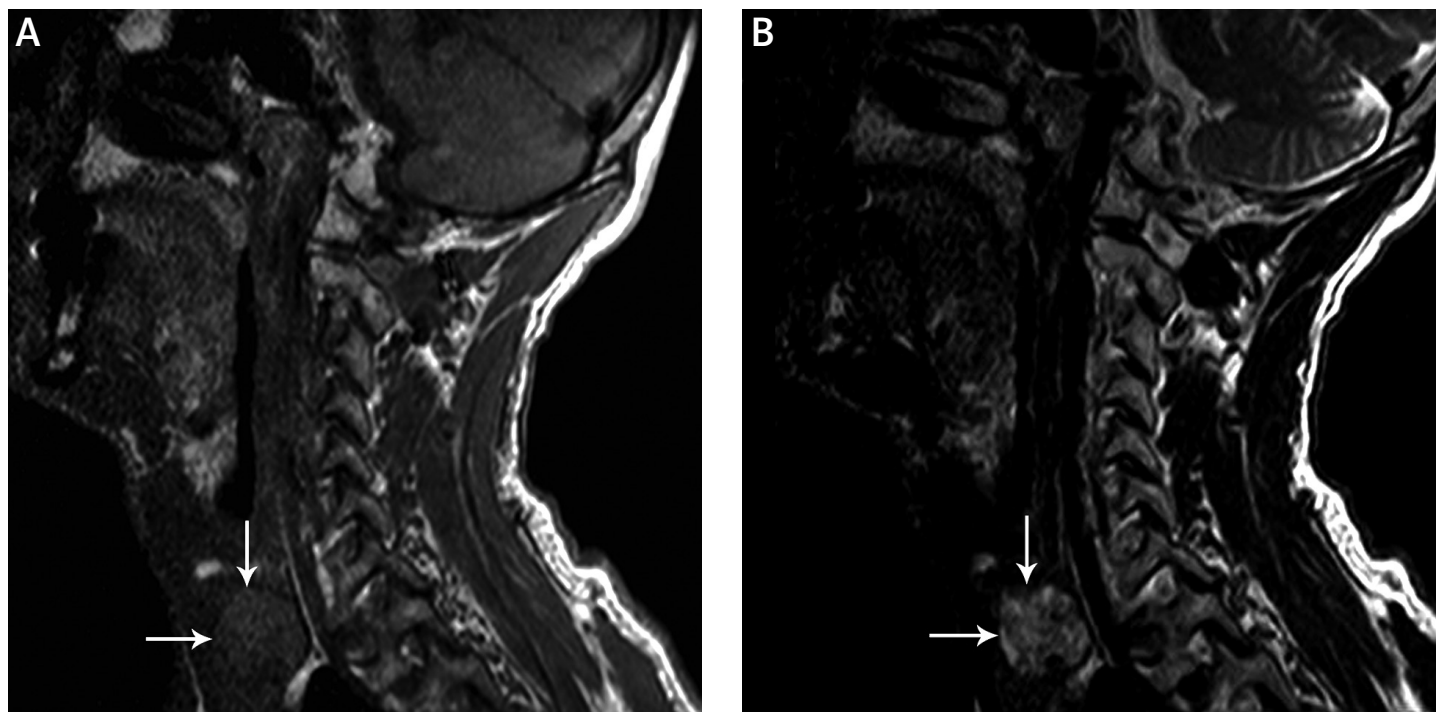

Figure 1. Nodule in the thyroid gland is hypointense on sagittal T1-weighted image (A) and hyperintense on sagittal T2-weighted image (B) (arrows)

SPSS software (version 16.0; SPSS Inc, Chicago, IL). The frequencies of incidental extraspinal pathological findings and congenital anomalies/anatomic variations were expressed as the number of cases/correspondent percentages and $95 \% \mathrm{Cl}$ for the percentages.

\section{Results}

In a total of 1106 MRI examinations, the percentages of incidental extraspinal pathological findings and congenital anomalies/anatomical variations were $16.6 \%(95 \% \mathrm{Cl}: 14.4-18.8)$ and $3.7 \%$ (95\% Cl: 2.6-4.3), respectively (Congenital anomalies/anatomic variations were detected only on lumbar spinal MRI). The percentage of incidental extraspinal pathological findings on all of the cervical spinal MRI $(n=237)$ was $25.7 \%(95 \% \mathrm{Cl}$ : 20.1-31.7). Thyroid nodules (Figure 1) were the most common incidental extraspinal pathological findings $(17.3 \%$ of all cervical spinal MRI, $95 \% \mathrm{Cl}$ : 12.4-22.1). The other incidental extraspinal findings on cervical spinal MRI are given in Table I. On all of the thoracic spinal MRI $(n=19)$, only inferior pole thyroid nodules could be demonstrated as incidental extraspinal pathological findings, with a percentage of $10.5 \%$ (95\% Cl: 9.6-11.5). On all of the lumbar spinal MRI $(n=850)$ and incidental pathological findings related to kidney, liver (Figure 2), internal genital organs (in females), and vascular systems were detected with a percentage of $14.2 \%$ (95\% Cl: 11.9-16.6), while the percentage of congenital anomalies/anatomical variations was $4.8 \%$ (95\% Cl: 3.4-6.3). Cortical and parapelvic renal cysts (Figure 3 ) were the most common incidental extraspinal pathological findings on all of the lumbar spinal MRI $(n=850)$, with a percentage of $6.2 \%(95 \% \mathrm{Cl}: 4.6-7.8)$. On lumbar spinal
MRIs of female patients $(n=558)$, uterine myomas (Figure 4) were the most common incidental extraspinal pathological findings related to their internal genital organs, with a percentage of 3.1\% (95\% Cl: 1.7-4.5). Incidental extraspinal congenital anomalies/anatomical variations were left renal vein variations including retroaortic left renal vein (RLRV) (Figure 5) and circumaortic left renal vein, retrovert uterus (in female patients), renal rotation anomalies, horseshoe kidneys (Figure 6), and kidney agenesis. Both incidental extraspinal pathological findings and congenital anomalies/ anatomical variations detected on lumbar spinal MRI are shown in Tables II and III, respectively.

During clinical evaluation and follow-up, fineneedle aspiration biopsy was performed in 9 out of 43 thyroid gland nodules of 41 patients. The cytopathological result in one case was suspicious, so surgery was performed. The histopathological result of this suspicious case was papillary carcinoma of the thyroid gland. Eventually, 6.5\% (95\% Cl: 2.6-9.4) of all cases with incidental extraspinal pathological findings underwent surgery, including renal cysts causing persistent

Table I. The distribution of incidental extraspinal pathological findings on 237 cervical spinal MRI examinations

\begin{tabular}{|lcc|}
\hline $\begin{array}{l}\text { Extraspinal pathological } \\
\text { findings }\end{array}$ & $\begin{array}{c}\text { Patients, } \\
n(\%)\end{array}$ & $\mathbf{9 5 \% ~ C l}$ \\
\hline Thyroid nodule & $41(17.3)$ & $12.4-22.1$ \\
\hline $\begin{array}{l}\text { Mucosal thickening } \\
\text { in paranasal sinuses }\end{array}$ & $11(4.6)$ & $1.9-7.3$ \\
\hline Tornwaldt cyst & $7(3.0)$ & $0.8-5.2$ \\
\hline Lipoma & $2(0.8)$ & $0.1-1.9$ \\
\hline Total & $61(25.7)$ & $20.1-31.3$ \\
\hline
\end{tabular}



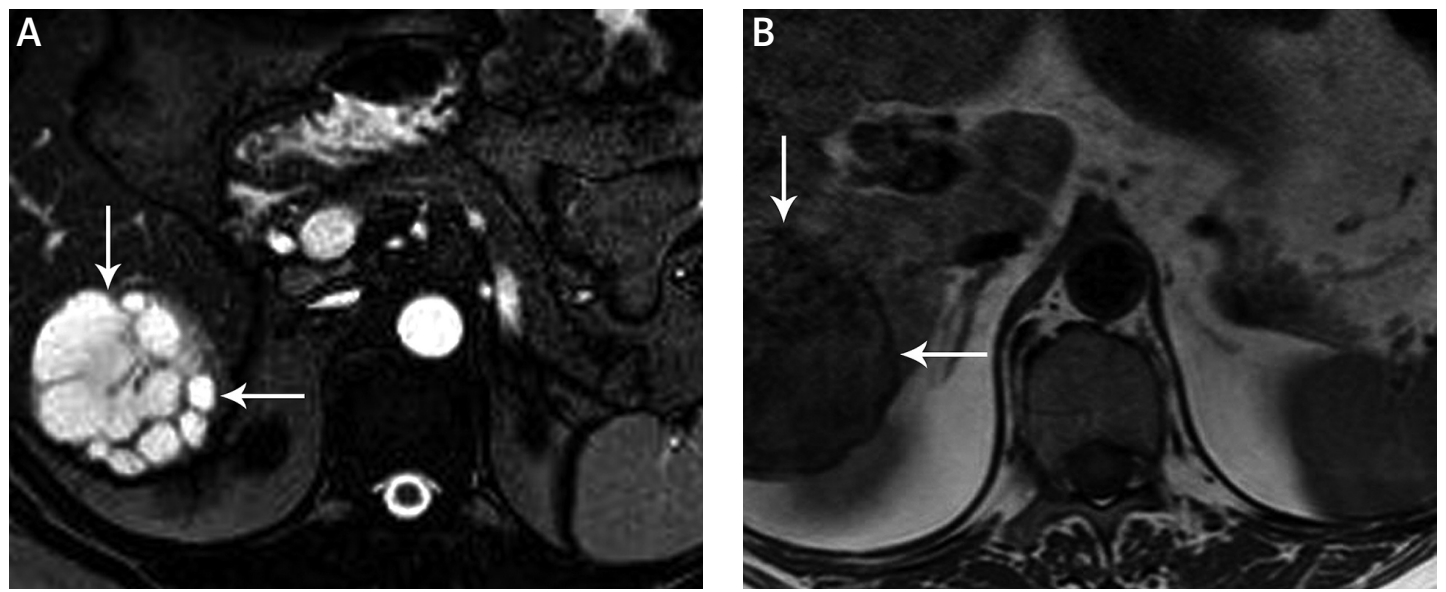

Figure 2. Lumbar MRI demonstrates hydatid cyst in the right lobe of the liver (arrows) on axial T2-weighted (A) and T1-weighted images (B), respectively

pain $(n=4)$, uterine myomas $(n=3)$, aneurysms of the abdominal aorta $(n=2)$ (Figure 7$)$, papillary carcinoma of the thyroid $(n=1)$, ovarian dermoid $(n=1)$, and recurrent renal cell carcinoma $(n=1)$. No complications developed in the surgically treated patients during hospitalisation, and they were discharged in good health.

\section{Discussion}

Chronic nonspecific neck or back pain is very common. Most normal connective tissues heal within 6-12 weeks unless instability or malignant or inflammatory tissue destruction is present. Therefore, in any prolonged pain, aetiologies should be ruled out. It is crucial to differentiate

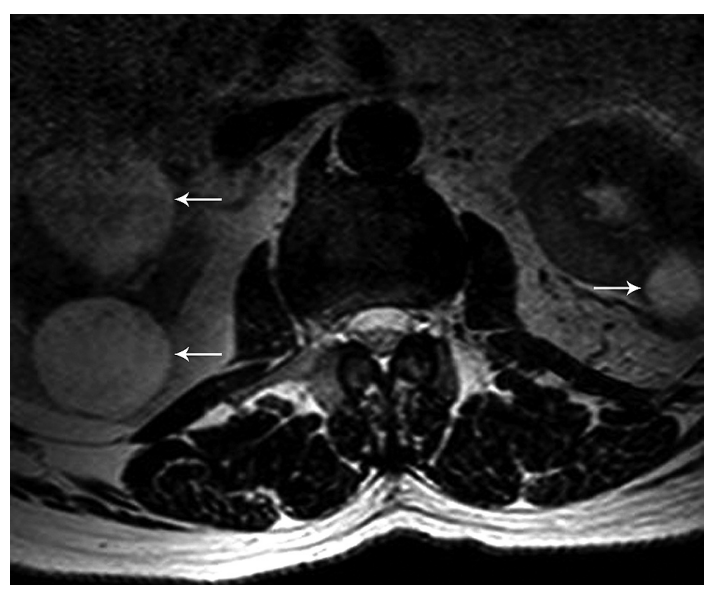

Figure 3. Lumbar MRI shows bilateral renal cysts (arrows) on axial T2-weighted TSE image
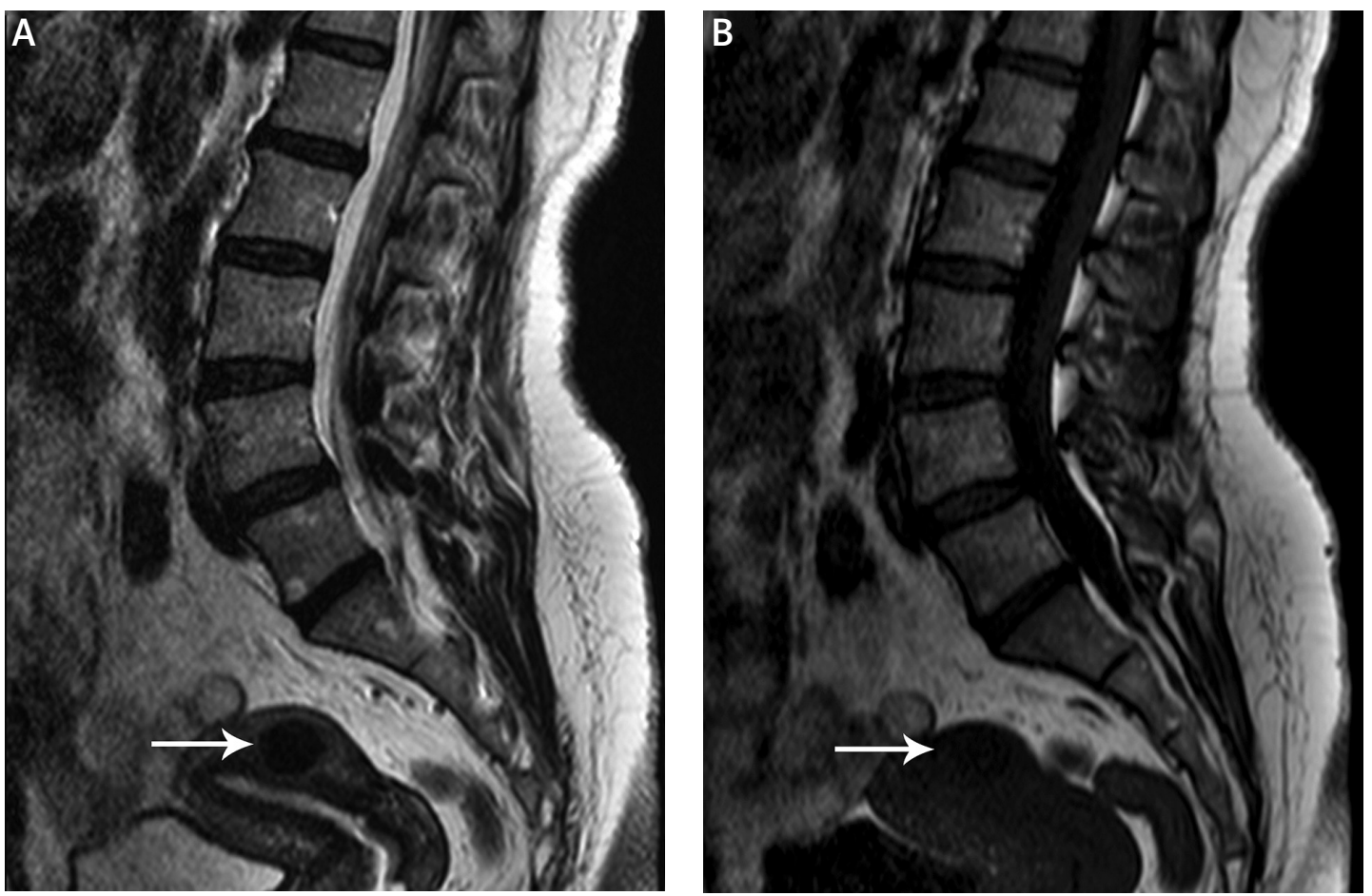

Figure 4. Lumbar MRI shows uterine myoma as hypointense lesion on sagittal T2-weighted (A) and T1-weighted images (B) (arrows) 


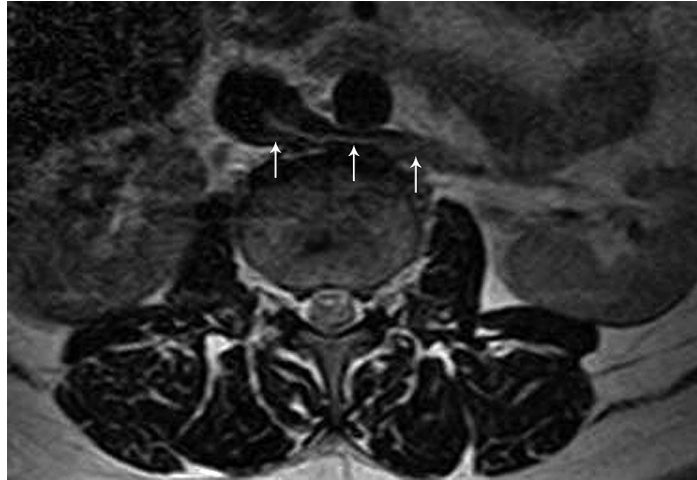

Figure 5. Lumbar MRI shows retroaortic left renal vein on axial T2-weighted image (arrows)

nonspecific pain from specific pain because the therapeutic techniques differ considerably. Computed tomography and MRI are the diagnostic tools for pain evaluation. In the literature there are a number of studies concerning incidental findings demonstrated with these modalities [10-14]. In the evaluation of the images of this study, we used PACS, which provides more information in the evaluation of images, increases efficiency, and decreases the time required for evaluation in radiology departments, and through the use of which the numbers of incidental findings and follow-up examinations have increased [15-17].

The most frequently observed incidental extraspinal pathologies on cervical spinal MRI are thyroid nodules [18], which was the case in the present study. The histopathological diagnosis of one such case who underwent surgery was papillary carcinoma of the thyroid. To our knowledge, in most institutes the patients are infrequently referred for thoracic spinal MRI examinations for the imaging of intervertebral discs, which is also true for our institute. Although we expected to find thoracic incidental extraspinal findings such as the ones reported in literature, including masses, pleural effusion, fibrous dysplasia and lymph-

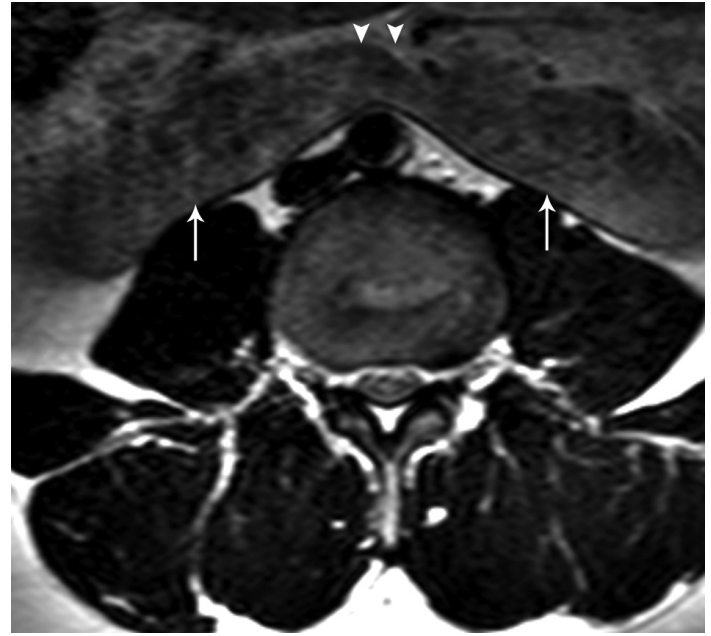

Figure 6. Lumbar MRI demonstrates horseshoe kidney on axial T2-weighted image (arrows: components of horseshoe kidney on right and left sides; arrowheads: connecting bridge of renal parenchyma anterior to abdominal aorta)

adenopathy, we found two inferior pole thyroid nodules [19]. Incidental extraspinal pathologies and congenital anomalies/anatomic variations are more frequent on MRI of the lumbar region because liver, ovaries, uterus, kidneys, and vascular structures are located in this region [17]. In two separate studies carried out using MRI of the lumbar spine, the rates of incidental findings were reported as $8.1 \%$ [16] and 8.3\% [17]. In the present study, this rate was $19 \%$ for all of the lumbar spinal MRI, which is higher than the studies reported previously. This might be due to the inclusion of all of the minor findings related to internal genitalia of females, such as nabothian cyst and all of the left renal vein variations. As was the case in the present study, renal cysts were reported to be the most frequent incidental pathological findings related with the urinary system in both males and females [20]. Dilli et al. identified RLRV and circumaortic left renal vein by using routine lumbar
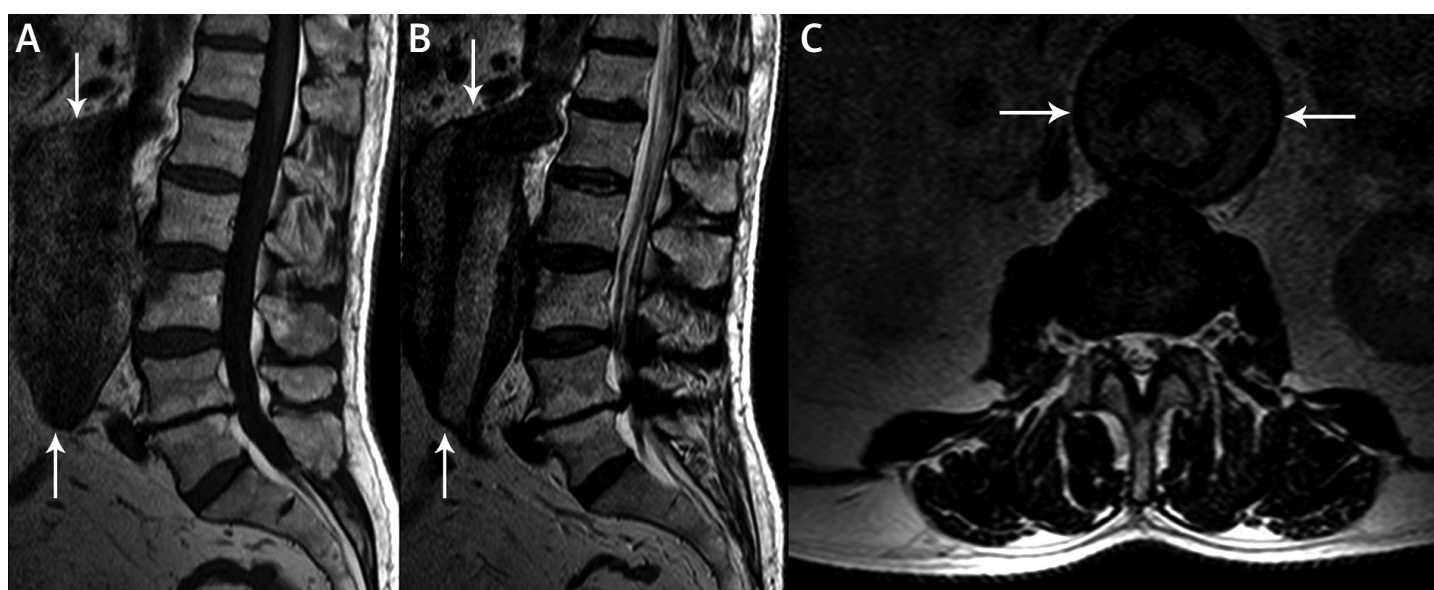

Figure 7. Lumbar MRI demonstrates aneurysm of abdominal aorta on sagittal T1-weighted (A), sagittal T2-weighted (B), and axial T2-weighted (C) images (arrows) 
Table II. The distribution of incidental extraspinal pathological findings on 850 lumbar MRI examinations (558 females, 292 males)

\begin{tabular}{|lcc|}
\hline $\begin{array}{l}\text { Extraspinal pathological } \\
\text { findings }\end{array}$ & $\begin{array}{c}\text { Patients, } \\
n(\%)\end{array}$ & $95 \% \mathrm{Cl}$ \\
\hline $\begin{array}{l}\text { Cortical and parapelvic } \\
\text { renal cyst }\end{array}$ & $53(6.2)$ & $4.6-7.8$ \\
\hline Uterine myoma & $17(3.1)$ & $1.7-4.5$ \\
\hline Ovarian cyst & $15(2.7)$ & $1.4-4.0$ \\
\hline Nabothian cyst & $7(1.3)$ & $0.4-2.2$ \\
\hline $\begin{array}{l}\text { Atrophic/hypoplastic renal } \\
\text { change }\end{array}$ & $9(1.1)$ & $0.2-2.0$ \\
\hline Subendometrial cyst & $5(0.9)$ & $0.1-1.7$ \\
\hline Hydronephrosis & $6(0.7)$ & $0.1-1.3$ \\
\hline $\begin{array}{l}\text { Hepatic masses } \\
\text { Aneurysm of abdominal } \\
\text { aorta }\end{array}$ & $2(0.2)$ & $0.1-1.1$ \\
\hline $\begin{array}{l}\text { Ovarian dermoid cyst } \\
\text { Recurrent renal cell }\end{array}$ & $1(0.2)$ & $0.1-0.5$ \\
\hline
\end{tabular}

spinal MRI examinations and reported that RLRV was the most frequent left renal vein variation, with a rate of $1.66 \%$ [21], which was also true for the present study (rate: $1.7 \%$ ). Many hepatic cysts and hepatic haemangiomas are found incidentally and do not require any treatment or further evaluation [22, 23]. In the present study, we detected hepatic masses including haemangiomas, simple liver cysts, and hydatid cyst in 5 cases. After detection of these lesions, our patients were followed-up without performing any interventional procedures, and no complications occurred. The most frequent pathologies incidentally detected in the female internal genital system in the present study were uterine myomas $(n=17)$, and three of these patients underwent surgery.

Some of the incidentally detected extraspinal findings have significant clinical importance and require immediate treatment. Early recognition and treatment of some incidentally detected urological pathologies, such as hydronephrosis, symptomatic renal cysts, and recurrent renal cell carcinoma, are important in order to avoid the long-term risk of renal damage. Aneurysm of abdominal aorta can cause complications that may be life-threatening [24]. We were able to incidentally diagnose some serious disorders in asymptomatic patients. In $1106 \mathrm{MRI}$ examinations we carried out, the percentage of incidental extraspinal pathological findings was $16.6 \%$. Of these patients, $6.5 \%(n=12)$ underwent surgery. The percentage of congenital anomalies/anatomical
Table III. The distribution of incidental extraspinal congenital anomalies/anatomic variations on 850 lumbar MRI examinations (558 females, 292 males)

\begin{tabular}{|lcc|}
\hline $\begin{array}{l}\text { Congenital anomalies/ } \\
\text { anatomic variations }\end{array}$ & Patients, $n$ (\%) & $95 \% \mathrm{Cl}$ \\
\hline $\begin{array}{l}\text { Left renal vein } \\
\text { variations }\end{array}$ & $\begin{array}{c}20(2.4) \\
\text { (retroaortic 1.7; } \\
\text { circumaortic 0.7) }\end{array}$ & $1.3-3.4$ \\
\hline Retrovert uterus & $10(1.8)$ & $0.9-2.7$ \\
\hline $\begin{array}{l}\text { Renal rotation } \\
\text { anomalies }\end{array}$ & $6(0.7)$ & $0.1-1.3$ \\
\hline Horseshoe kidney & $3(0.3)$ & $0.1-0.7$ \\
\hline Kidney agenesis & $2(0.2)$ & $0.1-0.6$ \\
\hline Total number of patients & $41(4.8)$ & $3.4-6.3$ \\
\hline
\end{tabular}

variations was $3.7 \%$. Although invasive procedures were performed for none of these cases up to now, radiological recognition of these congenital anomalies/anatomical variations is still clinically important since unawareness of them may cause severe haemorrhage and organ damage when a renal or retroperitoneal surgery is considered in the future $[21,25]$.

Whether or not reporting incidental extraspinal findings by the radiologists has positive or negative results in terms of patient health, reporting might cause unnecessary further examinations or might help save the patient's life, whereas not reporting may cause jeopardy in patient's health resulting in medical malpractice litigation [8]. We believe that it is one of the duties of the radiologist to report the incidental findings with scientifically sufficient differential diagnosis.

In any hospital where MRI is performed with an available PACS system or even with hard-copy archiving, any radiologist can retrospectively evaluate and analyse extraspinal incidental findings on the spinal MRI of their patients. As mentioned above, some studies related to incidental findings on MRI have already been performed and published [10, 15-17, 19, 21]. If we again perform a similar retrospective study in our hospital with similar numbers of patients, it is probable to get results close to those of the present study, although some different, minor results may also be obtained. Hence, despite its limitations, we believe that our study is reproducible. Since spinal MRI is directed towards only the intervertebral discs, axial images encompass solely the intervertebral disc interval. This, in turn, might have caused a lower than expected percentage of incidental findings. Also, we could increase the number of our patients by two or three fold in order to increase statistical power. Besides, cost-effectiveness is one of the issues that should be addressed, although we did not consider it in this study. 
In conclusion, on MRI examination of intervertebral discs, paying attention to incidentally detected pathological extraspinal findings and congenital anomalies/anatomical variations is very important due to the fact that they can alter the treatment of the patient or affect the patient's life. Therefore, they should be included in the reports since they will give additional and valuable information.

\section{Acknowledgments}

We are very grateful to Mr. Erdeniz Yurdakul and Mrs. Serpil Utku Gökbaş for their assistance in handling of the figures. We thank Hulya Melis Gulen for statistical analysis.

\section{References}

1. Liang CZ, Li H, Tao YQ, et al. The relationship between low $\mathrm{pH}$ in intervertebral discs and low back pain: a systematic review. Arch Med Sci 2012; 8: 952-6

2. Chou R, Qaseem A, Owens DK, Shekelle P. Clinical Guidelines Committee of the American College of Physicians. Diagnostic imaging for low back pain: advice for high-value health care from the American College of Physicians. Ann Intern Med 2011; 154: 181-9.

3. Troyanovich SJ, Harrison DD, Harrison DE. Low back pain and the lumbar intervertebral disk: clinical considerations for the doctor of chiropractic. J Manipulative Physiol Ther 1999; 22: 96-104.

4. Ly JQ. Systematic approach to interpretation of the lumbar spine MR imaging examination. Magn Reson Imaging Clin N Am 2007; 15: 155-66.

5. Berrington de González A, Mahesh M, Kim KP, et al. Projected cancer risks from computed tomographic scans performed in the United States in 2007. Arch Intern Med 2009; 169: 2071-7.

6. Mettler FA Jr, Thomadsen BR, Bhargavan M, et al. Medical radiation exposure in the U.S. in 2006: preliminary results. Health Phys 2008; 95: 502-7.

7. Golding SJ. Radiation exposure in CT: what is the professionally responsible approach? Radiology 2010; 255: 683-6.

8. Weiner DK, Kim YS, Bonino P, Wang T. Low back pain in older adults: are we utilizing healthcare resources wisely? Pain Med 2006; 7: 143-50.

9. Berlin L. The incidentaloma: a medicolegal dilemma. Radiol Clin North Am 2011; 49: 245-55.

10. Park HJ, Jeon $\mathrm{YH}$, Rho MH, et al. Incidental findings of the lumbar spine at MRI during herniated intervertebral disk disease evaluation. AJR Am J Roentgenol 2011; 196: 1151-5.

11. Rampinelli C, Preda L, Maniglio M, et al. Extrapulmonary malignancies detected at lung cancer screening. Radiology 2011; 261: 293-9.

12. Alpert JB, Naidich DP. Imaging of incidental findings on thoracic computed tomography. Radiol Clin North Am 2011; 49: 267-89.

13. Ding A, Eisenberg JD, Pandharipande PV. The economic burden of incidentally detected findings. Radiol Clin North Am 2011; 49: 257-65.

14. Frager DH, Elkin CM, Kansler F, Mendelsohn SL, Leeds NE. Extraspinal abnormalities identified on lumbar spine $\mathrm{CT}$. Neuroradiology 1986; 28: 58-60.
15. Kamath S, Jain N, Goyal N, Mansour R, Mukherjee K. Incidental findings on MRI of the spine. Clin Radiol 2009; 64: 353-61.

16. Wagner SC, Morrison WB, Carrino JA, Schweitzer ME, Nothnagel $\mathrm{H}$. Picture archiving and communication system: effect on reporting of incidental findings. Radiology 2002; 225: 500-5.

17. Green L. PACS: effect on incidental findings. Radiol Manage 2004; 26: 26-9.

18. Ezzat S, Sarti DA, Cain DR, Braunstein GD. Thyroid incidentalomas. Prevalence by palpation and ultrasonography. Arch Intern Med 1994; 154: 1838-40.

19. Olson EM, Wong WH, Hesselink JR. Extraspinal abnormalities detected on MR images of the spine. AJR Am J Roentgenol 1994; 162: 679-84.

20. Hélénon O, Correas JM, Balleyguier C, Ghouadni M, Cornud F. Ultrasound of renal tumors. Eur Radiol 2001; 11: 1890-901.

21. Dilli A, Ayaz UY, Karabacak OR, Tatar IG, Hekimoglu B. Study of the left renal variations by means of magnetic resonance imaging. Surg Radiol Anat 2012; 34: 267-70.

22. Washington K, Harris E. Masses of the liver. In: Sternberg's diagnostic surgical pathology. 5th ed. Mills EC, Carter D, Greeson JK (ed.). Lippincott Williams \& Wilkins Philadelphia 2009; 1192-2023.

23. Arbiser JL, Bonner MY, Berrios RL. Hemangiomas, angiosarcomas, and vascular malformations represent the signaling abnormalities of pathogenic angiogenesis. Curr Mol Med 2009; 9: 929-34.

24. Powell JT, Greenhalgh RM. Clinical practice. Small abdominal aortic aneurysms. N Engl J Med 2003; 348: 1895-901.

25. Thomas TV. Surgical implications of retroaortic left renal vein. Arch Surg 1970; 100: 738-40. 